\title{
The mycorrhizal fungi of Cymbidium promote the growth of Dendrobium officinale by increasing environmental stress tolerance
}

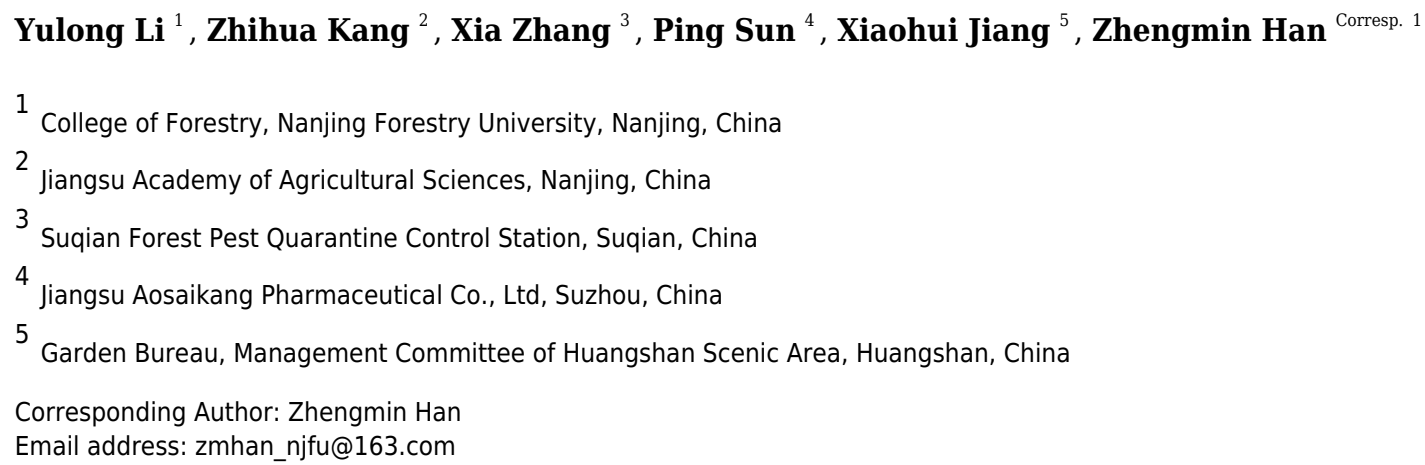

Dendrobium officinale is a medicinal herbal plant with important health care value and high demand. Due to its slow growth and scarcity in nature, its yield depends on intensified cultivation. During cultivation, biotic and abiotic stresses were important factors of production loss. In this study, the effects on $D$. officinale of four mycorrhizal fungi isolated from Cymbidium were tested. The obviously higher mass yield of the treated plants in medium and pots indicated the growth promotion effect of the fungi.

Furthermore, an abiotic stress test indicated stronger drought tolerance among the treated plants. For the biotic stress test, two root rot pathogens, Fusarium solani and Fusarium graminearum, were isolated and identified from root rot of $D$. officinale. In an in vitro inhibition test, the four mycorrhizal fungi could resist the growth of these pathogens. In vivo studies showed that these four mycorrhizal fungi could improve the survival rate and fresh weight and decrease the root rot rate of pathogen-inoculated seedlings. The four Cymbidium mycorrhizal fungi are compatible strains for improving the growth rate of $D$. officinale by increasing its environmental stress tolerance, providing an effective way to supply resources through artificial reproduction. 
1 The mycorrhizal fungi of Cymbidium promote the 2 growth of Dendrobium officinale by increasing 3 environmental stress tolerance

4 Yulong Li ${ }^{1}$, Zhihua Kang ${ }^{2}$, Xia Zhang ${ }^{3}$, Ping Sun ${ }^{4}$, Xiaohui Jiang ${ }^{5}$, Zhengmin Han ${ }^{{ }^{*}}$

5 'Department, Faculty, University name, Town, State

$6 \quad$ Department, Institution name, Town, State

$7 \quad{ }^{1}$ College of Forestry, Nanjing Forestry University, Nanjing, China.

8 2Jiangsu Academy of Agricultural Sciences, Nanjing, China

9 3Suqian Forest Pest Quarantine Control Station, Suqian, China.

10 4Jiangsu Aosaikang Pharmaceutical Co., Ltd, Nanjing, China.

$11{ }^{5}$ Garden Bureau, Management Committee of Huangshan Scenic Area, Huangshan, China

12 Corresponding author

13 Zhengmin Han

14 College of Forestry, Nanjing Forestry University, NO.59, Longpan road Nanjing, 210036, China. Email address: zmhan njfu@163.com

\section{Abstract}

Dendrobium officinale is a medicinal herbal plant with important health care value and high demand. Due to its slow growth and scarcity in nature, its yield depends on intensified cultivation while biotic and abiotic stresses were important factors that causes production loss. Orchidaceae can form association with rhizoctonias collectively, and studies have found that some orchids showed a high level of strain-species specificity to orchid mycorrhizal fungi (OMF), yet the specificity of OMF on D. officinale needs to explored. In this study, the effects on D. officinale of four OMF isolated from Cymbidium were tested. The obviously higher mass yield of the treated plants in medium and pots indicated the growth promotion effect of the fungi. Furthermore, an abiotic stress test indicated stronger drought tolerance among the treated plants. For the biotic stress test, two root rot pathogens, Fusarium solani and Fusarium graminearum, were isolated and identified from root rot of $D$. officinale. In an in vitro inhibition test, the four OMF could resist the growth of these pathogens. In vivo studies showed that these four OMF could improve the survival rate and fresh weight and decrease the root rot rate of pathogen-inoculated seedlings. The four OMF namely; Hyphomycete sp., Umbelopsis sp., Ceratorhiza sp. and Ceratorhiza sp. are compatible strains for improving the growth rate of $D$. officinale by increasing its environmental stress tolerance, providing an effective way to supply resources through artificial reproduction.

\section{Introduction}

Dendrobium officinale Kimura et Migo belongs to Orchidaceae and has high medicinal value. It has been widely used in Asian countries for hundreds of years to enhance immunity, provide antithrombotic and antineoplastic effects, and delay ageing[1-4]. Due to the rising demand for this herb, wild $D$. officinale is severely exploited and will become extinct. Currently, 
40

41

42

43

44

45

46

47

48

49

50

51

52

53

54

55

56

57

58

59

60

61

62

63

64

65

66

67

68

69

70

71

72

73

74

75

76

77

78

79

massive amounts of $D$. officinale are rapidly propagated under intensified cultivation to overcome this problem[5]. However, issues in artificial cultivation have also emerged, such as

low growth rate, poor adaptability and plant disease. Notably, susceptibility to withering and root and stem rot mainly caused by fungal disease has resulted in serious economic losses in the cultivation of this plant $[6,7]$.

Mycorrhizal fungi are a group of fungi that live in healthy plants at a certain or all life stages $[8,9]$. Mycorrhizal fungi can form a mutually beneficial relationship with host plants. Orchid mycorrhizal fungi (OMF) are present in all orchid species and play an important role in the growth of orchid species by establishing obligate relationships conducive to gain carbon, nutrients and water, especially during early life stages when orchids lack sufficient nutrient reserves in seeds [10,11]. While orchid acquires nutrients by mycoheterotrophy, the root function of the host can be strengthened by the OMF to increase the absorbability of mineral elements, especially phosphorus [12]. OMF can also stimulate the growth and development of host plants, improving the host's resistance to stress [13,14]. Applying suitable OMF could be advantageous for orchid conservation and reproduction, e.g., propagation, ex situ seeding or germination. However, the acceptability of and preference for OMF by the host is an important factor to consider before application [15-17]. Mycorrhizal fungi may have different effects on different hosts, which may depend on the process of recognition between the host and mycorrhizal fungus as well as compliance with symbiosis [18-20]. Suitable mycorrhizal fungi are needed for seed germination and seedling growth of $D$. officinale under natural conditions [21, 22]. Even though adequate nutrition is provided by artificial cultivation, this plant is slow-growing and sensitive to biological and abiotic stressors. This is probably because appropriate mycorrhizal fungi are deficient in artificial cultivation conditions.

Root rot is a major disease of $D$. officinale that is mainly caused by fungal pathogens. It can weaken the root function of plants until the whole plant dies [23]. This disease can cause massive infection and death in intensive cultivation of $D$. officinale [6, 7]. Fungal pathogens in Dendrobium have been identified, such as Fusarium sp., Pythium sp. and Ceratobasidium sp. $[6,7,24]$. Chemical agents such as metalaxyl-M, propamocarb, and Bordeaux mixtures have been widely used to prevent and treat this disease [25]. However, chemical residues on the products inhibit the growth of probiotic fungi and lead to low-quality products that are harmful to sustainable agricultural development [26, 27].

In view of the above problems in intensified cultivation, four OMF namely; GDB162, GDB254, GS222, MLX102 isolated from other Cymbidium were used to promote the growth and to increased abiotic and biotic stress tolerance of $D$. officinale because these strains reportedly showed growth promotion effect on Cymbidum such as C. hybrid, C. goeringii, C. mastersii in former studies [28-30]. On the other side, the pathogenic fungi isolated and identified due to the root disease is still a problem in intensified cultivation of $D$. officinale $[6,7]$. Further, these OMF were used to prevent and control root rot $D$. officinale. Our findings have great significance in increasing and reducing the morbidity of $D$. officinale under intensive cultivation.

\section{Materials \& Methods}


80

81

82

83

84

85

86

87

88

89

90

91

92

93

94

95

96

97

98

99

100

101

102

103

104

105

106

107

108

109

110

111

112

113

114

115

116

117

118

119

\section{Plant materials, fungi and medium}

The four mycorrhizal fungi are GDB162 (Hyphomycete sp.) [30], GDB254 (Umbelopsis sp.) [30], GS222 (Ceratorhiza sp.) [30, 31] originating from Cymbidium faberi Rolfe in Anhui Province, China, and MLX102 (Ceratorhiza sp.) [28-30], which was isolated from Cymbidium sinense (Jackson ex Andrews) Willd in Yunnan Province, China. D. officinale with root disease was collected from a nursery in Wujiang city, Jiangsu Province, China. Fungi were incubated on PSA medium containing $20 \mathrm{~g}$ sucrose, $20 \mathrm{~g}$ agar, $200 \mathrm{~g}$ potato and $1000 \mathrm{~mL}$ water. Mycorrhizal fungal infection of $D$. officinale was facilitated by $\mathrm{DE}$ symbiotic medium [32] containing $\mathrm{K}_{2} \mathrm{SO}_{4}$ $1.0 \mathrm{mg} / \mathrm{L}, \mathrm{FeSO}_{4} 100 \mathrm{mg} / \mathrm{L}, \mathrm{MnCl}_{2} 3.3 \mathrm{mg} / \mathrm{L}, \mathrm{NaMoO}_{4} 1.0 \mathrm{mg} / \mathrm{L}, \mathrm{MgSO}_{4} 0.5 \mathrm{mg} / \mathrm{L}, \mathrm{KH}_{2} \mathrm{PO}_{4} 0.4$ $\mathrm{mg} / \mathrm{L}, \mathrm{H}_{3} \mathrm{BO}_{4} 25 \mathrm{mg} / \mathrm{L}, \mathrm{ZnSO}_{4} 2.8 \mathrm{mg} / \mathrm{L}$, yeast extract $10 \mathrm{~g} / \mathrm{L}, 9 \mathrm{~g} / \mathrm{L}$ soluble starch and $4.5 \mathrm{~g} / \mathrm{L}$ agar powder ( $\mathrm{pH}$ 6.0). Murashige and Skoog (1962) (MS) medium was used as a medium for the growth of $D$. officinale seedlings[33]. The rooting medium was MS medium plus $1 \mathrm{mg} / \mathrm{L}$ NAA (1-naphthylacetic acid).

\section{The plant effects of mycorrhizal fungi on $D$. officinale}

D. officinale PLBs were cultured in rooting medium. After that, three seedlings with similar growth conditions (approximately $1.5 \mathrm{~g}$ of each) were transferred to solid DE symbiotic medium and cultured at $25^{\circ} \mathrm{C}$ for two days to verify the absence of contamination. Three $5 \mathrm{~mm}$ diameter blocks of agar containing fungi were evenly inoculated around $D$. officinale seedlings. For the testing of tissue culture seedlings, the seedlings were cultured at $25^{\circ} \mathrm{C}$ and $12 \mathrm{~h}$ daily light for 60 days. Then, fresh weight, chlorophyll content, stem polysaccharide levels, and root acid phosphatase activity (ACP) were measured and analyzed. For the testing of pot seedlings, the seedlings were transplanted to the pots after 15 days of OMF inoculation and were grown in a closed green house with natural light at $25^{\circ} \mathrm{C}$ for 5 months. Then, the survival rate, stem length, stem diameter and leaf number were measured and analyzed. The experiment was performed in thirty replicates.

\section{The effect of mycorrhizal fungi on plant drought resistance}

$D$. officinale seedlings in pot with similar growth condition and weight were selected for testing of drought resistant. The 4 leaves (about $5 \mathrm{~mm}$ around wide) of $D$. officinale infected by mycorrhizal fungi were placed around the root of the seedling for mycorrhizal fungi inoculation and the CK group consist of leaves without mycorrhizal fungi incubation [34, 35]. The mycorrhizal fungi infected the root of the plant and adequate water was supplied. Then, three experimental groups were set up. The group I (CK) was adequately water was supplied every week for the whole time. The group II was subjected to limited water supply every week, and the group III was subjected to adequate water was supplied only once at the beginning. After that, the survival rate of the plant of group III was recorded at 15, 30, 60 days and the MDA content of group I and II were measured and analyzed at 60 days. Each group had 10 replicate. Malonaldehyde (MDA) content was determined with the method of thibabituric acid (TBA) by using an MDA assay kit (Nanjing Jiancheng Bioengineering Institute, Nanjing, China, cat: A0031-2) according to the manufacturer's instructions and determined at $532 \mathrm{~nm}$. The experiments were performed in triplicate. 
120

121

122

123

124

125

126

127

128

129

130

131

132

133

134

135

136

137

138

139

140

141

142

143

144

145

146

147

148

149

150

151

152

153

154

155

156

157

\section{Determination of chlorophyll, and phosphatase activity}

Chlorophyll $a$ and $b$ were determined as described previously [36]. Fragmented fresh leaves $(0.25 \mathrm{~g})$ were transferred into a mortar, and then $2.5 \mathrm{ml}$ of acetone was added. The mixture was ground to a homogenate, and $2.5 \mathrm{ml}$ of $80 \%$ acetone was added. The samples were centrifuged at $12,000 \times \mathrm{g}$ for $10 \mathrm{~min}$. The volume of the supernatant was adjusted to $10 \mathrm{ml}$ with $80 \%$ acetone. Then, $0.5 \mathrm{ml}$ chlorophyll extract was diluted with $2 \mathrm{ml}$ of $80 \%$ acetone, and the solution was transferred to a cuvette. The $80 \%$ acetone solution was used as a control. The absorbance was measured by a spectrophotometer at 645 and $663 \mathrm{~nm}$ to determine the chlorophyll $a$ and b contents, respectively.

Acid phosphatases activity was assayed using $\rho$-nitrophenol. Fresh root $(0.5 \mathrm{~g})$ was transferred to a mortar and pestle at $0-4^{\circ} \mathrm{C}$ in $50 \mathrm{mM}$ sodium acetate buffer ( $\mathrm{pH}$ 5.5). The sample were centrifuged at $12000 \mathrm{~g}$ for $15 \mathrm{~min}$ and the supernatant collected. ACP was determined using $\rho$-nitrophenol phosphate as substrate and measuring the amount of $\rho$-nitrophenol produced. Activity was quantified by comparing the absorption at $410 \mathrm{~nm}$ to a standard curve of diluted $\rho$ nitrophenol solutions and $\mathrm{NaOH}$. One unit of phosphatase is equivalent to the amount of enzyme producing $1 \mu \mathrm{M}$ of product per min under assay conditions.

\section{Pathogen isolation and identification}

The pathogens were isolated from rotted roots of $D$. officinale. The rotted roots were soaked in $70 \%$ ethanol for $5-10$ seconds, then in $0.1 \%$ mercury bichloride for 90 seconds and washed with sterilized water three times. The treated roots were incubated on PSA medium at $26^{\circ} \mathrm{C}$ for $3-5$ days. All suspected mycelium tips of pathogens were transferred to new PDA medium. The following three primer pairs were used to amplify the fungal rRNA internal transcribed spacer (ITS), the second largest RNA polymerase subunit (RPB2) and large fragment of ribosomal (LSU) fragments: ITSF (5'- TCCGTAGGTGAACCTGCGG-3')/ ITSR (5'TCCTCCGCTTATTGATATGC-3') [37], RPB2-5F2(5' - GGGGWGAYCAGAAGAAGGC)/7cR (5' - CCCATRGCTTGYTTRCCCAT) [38] and LSUF LSUF (5'- ATCCTGAGGGAAACTTC-3')/ LSUR (5' - GTACCCGCTGAACTTAAGC-3') [39].

\section{Pathogenicity test}

A pathogenicity test was performed on $D$. officinale by following Koch's postulates with tissue culture seedlings and plants in pots. The seedlings were incubated on DE medium for 2 weeks at $26^{\circ} \mathrm{C}$ with a 12-h dark/light cycle. The pathogenic fungi were evenly inoculated around seedlings for 30 days of co-incubation. The plants in pots containing dried bark and peat were grown at a ratio of $2: 5$ at $26^{\circ} \mathrm{C}$ for 15 days. The fungi were incubated in Czapek medium with $120 \mathrm{rpm}$ shaking and $25^{\circ} \mathrm{C}$ for 4 days. Then, the conidia suspensions $\left(10^{6} \mathrm{CFU} / \mathrm{ml}\right)$ were created and inoculated into the rhizosphere of plants in pots. The plants were incubated at $26^{\circ} \mathrm{C}$ and $65 \%$ relative humidity for 30 days, and the root rot rate and death rate were recorded. The root rot severity was rated on a scale of 1 (root no wilting, white and plump) to 5 (plant death, root complete yellow and shrinking) [40]. The experiments were performed in triplicate. 
158

159

160

161

162

163

164

165

166

167

168

169

170

171

172

173

174

175

176

177

178

179

180

181

182

183

184

185

186

187

188

189

190

191

192

193

194

\section{Antagonism studies in vitro}

The mycorrhizal fungi and pathogens were inoculated on PSA medium $(2 \mathrm{~cm}$ away from the edge in a $9 \mathrm{~cm}$ petri dish) at an interval of $5 \mathrm{~cm}[41,42]$. The inoculated plate was incubated at $20^{\circ} \mathrm{C}$ for 1 week, and the colony diameters of both fungi were measured every 24 hours. The experiments were performed in triplicate.

\section{The effect of mycorrhizal fungi on pathogens}

Three $5 \mathrm{~mm}$ diameter blocks of agar containing OMF were evenly inoculated around $D$. officinale seedlings respectively. Then, the seedlings were incubated at $25^{\circ} \mathrm{C}$ under $12 \mathrm{~h}$ daily light for 15 days. After that, the co-incubated seedlings were transferred to pots in a greenhouse, and the seedlings were inoculated with $1 \mathrm{~mL}$ of the conidia suspensions $\left(10^{6}\right.$ $\mathrm{CFU} / \mathrm{ml}$ ) of the pathogenic fungi. The survival rate, total root rot and flesh weight were recorded after 30 days. Ten replicates of the experiment were performed.

\section{Statistical analysis}

Statistical analyses were performed using SPSS 17.0. One-way analysis of variance (ANOVA) and Tukey's test were used to detect the differences in seedling weight, polysaccharide rate, chlorophyll content, acid phosphatase activity, MDA content, colony diameter and root rot rate, survival rate.

\section{Results}

\section{The promotion of growth of tissue-cultured $D$. officinale by mycorrhizal fungi}

Four mycorrhizal fungi were inoculated to test their growth-promoting effect on tissuecultured $D$. officinale. The results showed that these four strains promoted the growth of $D$. officinale for 60 days. The seedlings demonstrated better growth, with bright green shoots (Fig. 1), higher fresh seeding weight (Fig 2A), higher chlorophyll contents (Fig 2B), and higher levels of polysaccharide in the stems (Fig 2C) compared with those in the control group (CK). The differences between the stimulatory effects of GS222, GDB254, and MLX102 on the pigment contents were not significant. MLX102 had the strongest promoting effect on chlorophyll a levels, which demonstrated a 1.85-fold increase compared to that in the CK. GDB254 was the most effective stimulator of chlorophyll b (2.72-fold) and total chlorophyll (1.94-fold) contents compared to those in the CK. GDB254-treated plants had a greater increase in weight. The acid phosphatase activity (Fig 2D) indicated that the phosphorus absorption of inoculated $D$. officinale roots was weaker than that in the CK roots.

\section{The promotion of growth of $D$. officinale in pots by mycorrhizal fungi}

To study the promoting effect of these mycorrhizal fungi on $D$. officinale, four fungi were inoculated into cultivated seedlings in pots. The results showed that all the mycorrhizal fungi had a growth-promoting effect on the seedlings after for 5 months. The seedling survival rate after transplantation from the medium to the substrate in the GDB254, MLX102, GS222, and GDB162 
195

196

197

198

199

200

201

202

203

204

205

206

207

208

209

210

211

212

213

214

215

216

217

218

219

220

221

222

223

224

225

226

227

228

229

230

231

232

groups was increased by approximately $16 \%, 13 \%, 10 \%$, and $13 \%$, respectively, compared with that in the CK group (Fig. 3A). The stem diameter, stem height, and leaf number of the seedlings inoculated with mycorrhizal fungi (Fig. 3 B, C, D) were significantly increased. GDB162 had the most efficient growth-promoting effect of all mycorrhizal fungi and induced an increase in seedling thickness, height, and leaf number by approximately $118 \%, 54 \%$, and $58 \%$, respectively.

\section{Drought stress resistance of $D$. officinale due to mycorrhizal fungi}

To evaluate the effect of these fungi on the drought resistance of $D$. officinale, the survival rate and MDA (malondialdehyde) content were measured under various drought conditions. The results showed that the survival rate of $D$. officinale was significantly improved in the $15,30,60$ days of drought conditions in the presence of mycorrhizal fungi (Fig. 4A). In the GDB162, GS222, and GDB254 tested groups, the survival rate of $D$. officinale at various drought periods was improved by more than $40 \%$ compared with the CK group. On the other hand, the MDA content in mycorrhizal fungi inoculation groups was steadier and maintained at a low level in the drought period, while the MDA content of the CK group during drought is increased to twice times that of the normal growth environment. This phenomenon was more obvious in the MLX102 and GDB254 groups than in the other groups (Fig. 4B). In addition, most of seedlings in group III were dead, hence, MDA content analysis was not conducted for this group. The results indicated that the four mycorrhizal fungi could promote drought resistance in $D$. officinale.

\section{Isolation and identification of pathogenic fungi}

Two fungi, TS1 and TS2, were isolated from $D$. officinale with root rot and showed pathogenicity (Figure 5). The sequence data of TS1 showed $100 \%$ homology to Fusarium solani (GenBank accessions ITS-MT638068.1 (449/449 bp), RPB2-MK606410.1 (861/861 bp), LSUMT533257.1 (726/726 bp), and the pathogen was identified as Fusarium solani. Sequence data of TS2 showed homology to Fusarium graminearum (GenBank accessions ITS-KU254606.1 (789/789 bp), RPB2-LT222053.1 (970/976 bp), LSU-MH877271.1 (857/857 bp), and the pathogen was identified as Fusarium graminearum.

\section{Pathogenicity test of the pathogens}

TS1 and TS2 show pathogenic effects on D. officinale in medium (Figure S1) and pot conditions. The pathogen-incubated groups showed root rot symptoms after two weeks (Fig. 6), while the roots of the control group were healthy and strong. TS1 was strongly pathogenic; most seedling's root turned yellow and withered while the survival rate of the TS2 group was $55 \%$, and the roots turned yellow and black (Fig. 6 B, C).

\section{Competitiveness of the four mycorrhizal fungi with pathogenic fungi}

To test the interaction of four mycorrhizal fungi and pathogens, these strains were used to confront these pathogens in vitro. All the pathogenic fungi grew with mycorrhizal fungi on the medium, but the pathogens had a faster growth rate. The growth of TS1 was significantly delayed under the effect of mycorrhizal fungi (Fig. 7 A-D). The growth curve indicated that the 
233

234

235

236

237

238

239

240

241

242

243

244

245

246

247

248

249

250

251

252

253

254

255

256

257

258

259

260

261

262

263

264

265

266

267

268

269

270

271

272

273

274

275

276

growth rate of TS1 was obviously slowed down in 3-5 days when it came in close proximity to the mycorrhizal fungus. The inhibition of mycorrhizal fungi on TS2 was not as obvious as that on TS1, in which colonies touched each other (Fig. 7 E-H).

\section{Mycorrhizal fungi improve root rot resistance of $D$. officinale}

The competitive effect between the mycorrhizal fungi and pathogens in vitro did not completely support the biocontrol potential of the mycorrhizal fungi in the hosts. Thus, we evaluated the potential of mycorrhizal fungi as biocontrol agents against pathogens in $D$. officinale on DE medium and in pots in greenhouses via interactions among hosts, mycorrhizal fungi, and pathogens together. The survival rate of $D$. officinale indicated that four mycorrhizal fungi played roles in improving the survival ability of tissue culture seedlings (Fig. 8). The highest survival rate $(\sim 50 \%$ in TS1 and $\sim 80 \%$ in TS2) indicated that MLX102 supplied stronger disease resistance for $D$. officinale. The mycorrhizal fungus increased the survival rate to a limited extent $(0 \sim 20 \%)$ due to the weaker lethality of TS2 (Fig. 8A). The root rot rate and fresh weight of $D$. officinale in the greenhouse both indicated improved disease resistance to pathogenic fungal infection. The root rot rate of the CK groups (>90\%, Fig. 8 B) indicated that both pathogenic fungi were highly virulent. MLX102 inhibited the root rot rate caused by TS1 and TS2 with an efficiency of approximately $40 \% \sim 50 \%$ (Fig. 8). GS222 seemed unable to inhibit root rot caused by TS1, but it had a certain effect on TS2, with an inhibition rate of approximately $25 \%$. The other two mycorrhizal fungi had more moderate inhibition effects, with inhibition efficiencies between those of MLX102 and GS222. All the mycorrhizal fungi led to an increase in fresh weight to some extent. Although the reduction in root rot rate did not correspond to an increase in fresh weight, all the different mycorrhizal fungi have the effect of reducing the root rot rate and increasing the fresh weight (Fig. $8 \mathrm{C}$ ).

\section{Discussion}

Mycorrhizal fungi are important resources for $D$. officinale growth promotion and intensify their tolerance to the environment [43], with promising agricultural applications [44, 45]. But the degree of specificity of most orchids to their mycorrhizal associates remains unknown. In this study, we have shown that mycorrhizal fungi of Cymbidium could promote the growth of $D$. officinale tissue culture seedlings and pot cultivation seedlings. Interestingly, mycorrhizal fungi improved the adaptation of $D$. officinale to biotic stress and abiotic stress resistance. It is important to note that the mycorrhizae from Cymbidium are compatible with $D$. officinale. Literatures have shown that mycorrhizal fungi can promote the growth of orchids as well as stimulation of germination [46-48]. However, few studies employed orchid-mycorrhizal fungi to promote the growth of $D$. officinale. Moreover, we also found and identified the root rot pathogen (soil-borne) of $D$. officinales and mycorrhizal fungi could increase such biotic stress tolerance. This finding will improve our understanding of the promotion effects of OMF on $D$. officinale.

According to reports, OMF have contributed to the growth, development, nutrient uptake, and resistance to pathogenic infection of different plant species. These four fungi have been proven that OMF could promote growth of orchids such as C. hybrid, C. goeringii, C. mastersii in former studies [28-30]. In this study, we were able to prove that OMF significantly contributed to both physiological and morphological parameters of $D$. officinales expressing different growth effect on $D$. officinale. The stem length, diameter and polysaccharide levels all increased, indicating that the quality of the seedlings was improved. 
277

278

279

280

281

282

283

284

285

286

287

288

289

290

291

292

293

294

295

296

297

298

299

300

301

302

303

304

305

306

307

308

309

310

311

312

313

314

315

316

317

318

319

320

The biochemical index quantificationally also demonstrated the promotion effect of these fungi. Our results show that all four mycorrhizal fungi could increase the weight as well as improve the content of photopigments (chlorophyll a, chlorophyll b and total chlorophyll), on average. The increase of chlorophyll in the symbiotic plant probably resulted in higher photosynthetic rates and thus improved plant biomass. The chlorophyll b content of $D$. officinale in the medium was especially obvious. As an index of blue and violet light absorption, the high chlorophyll b content indicated that mycorrhizal fungi could promote low light capacity utilization [49]. This increase of chlorophyll content by mycorrhizal fungi inoculation may be due to an increase in stomatal conductance, photosynthesis, transpiration [50, 51].

AcPase activity describes the phosphorus usage efficiency of plants. The decreasing phosphatase efficiency in the mycorrhizal fungi coculture groups indicated that adequate phosphorus was supplied. The OMF supplied the orchid with phosphorus $(\mathrm{P})$ demonstrating that the OMF was involved in P uptake and transfer in former studies $[52,53]$, which could provide additional phosphorus to $\mathrm{P}$ acquired by the root of host plant. Similar studies of OMF proves that $P$ absorption and transfer abilities could provide phosphorus for host plant usage [54, 55]. Furthermore, difference in ACPase activity could be attributed to the metabolism of plant host during interaction with OMF where the cell of OMF is digested in cavity and the activity occurs with some level of variation $[31,56]$. In addition, the AcPase activity of root could be different in root after different mycorrhizal fungi species invade the host plant [57].

OMF could enhance the absorption ability of the root system as well as the hydraulic conductivity of plants, which is associated with drought tolerance $[58,59]$.The increased survival rate and decreased MDA content under drought conditions indicated that OMF increased the drought tolerance of $D$. officinale. The potential of the mycorrhizal fungi to confer drought resistance to host plants is important for plant adaptation to the environment [60,61].

Root rot caused by pathogenic fungi has led to great economic losses in the production of D. officinale and orchids [23, 62, 63]. Two pathogenic fungi were confirmed: TS1 and TS2 were Fusarium solani and Fusarium graminearum, respectively, while the severe root rot in crops and fruits could be caused by these fungi [23, 62, 64-68]. Fusarium species are one of the most common soil-borne pathogens for plants and are causal agents of orchid root rot [69, 70]. These pathogens are major problems for commercial orchid production [69, 71].

These pathogens also caused root rot in D. officinale in this study. TS1 is a highly pathogenic fungus that causes a high lethal ratio under sterile medium conditions. However, the seedling roots tended to rot in the pot after being inoculated with this pathogen. This pathogen is relatively more likely to cause a high rate of root rot in greenhouses. This difference may be because the DE medium is also suitable for fungal growth. It provides fungi with adequate nutrients, which means that it helps pathogen growth and exerts virulence [72, 73]. The four mycorrhizal fungi in this study exhibited resistance to pathogens on plates and pots, which was mainly manifested both in the increased survival rate of seedlings and in reduced root rot of the plants. They exhibited different inhibition zone diameters in the plate competition test, which may be caused by antibiotics and lytic enzymes produced by the fungi that has reported in other studies of mycorrhizal fungi [74-76]. These effects of the mycorrhizal fungi of $D$. officinale are essential to the resistance of root rot pathogenic fungi in production.

Biological control has advantages, such as relatively few side effects, less pollution and longer effective times, and is a major trend in the development of agriculture and forestry today 
$321[44,77]$. This is an effective biocontrol method that is widely used in farming [78-80], and it is 322 also suitable for use in the production of $D$. officinale today. Mycorrhizal fungi could be important 323 biocontrol resources for competing with and inhibiting the reproduction of the pathogens by

324 producing antibiotic or antifungal compounds and stimulating plant defence responses [81, 82]. 325 Abiotic stress could weaken plant defences and enhance pathogen infection probability [83]. It 326 has been suggested that the combination of abiotic and biotic stress could cause increased 327 serious disease [84, 85]. Mycorrhizal fungi could enhance resistance to both stresses, 328 indicating that these fungi are useful resources for the growth promotion of $D$. officinale.

\section{Conclusions}

330 Together, the mycorrhizal fungi in the culture medium or the cultivated substrate could 331 promote the production of $D$. officinale by increasing resistance to biological and abiotic stress. 332 Furthermore, we isolated and characterized two fungal root pathogens of $D$. officinale. Inhibition 333 of fungal plant pathogens by mycorrhizal fungi isolated from different Cymbidium was a suitable means to control the disease caused by Fusarium. This finding is important for growth promotion, root rot disease prevention and control in $D$. officinale production. 


\section{References}

338

339

340

341

342

343

344

345

346

347

348

349

350

351

352

353

354

355

356

357

358

359

360

361

362

363

364

365

366

367

368

369

370

371

372

373

374

375

376

377

378

379

380

381

382
1.Zhao, Y., L. Xu, and Y. Hua, Effects of Dendrobium officinale polysaccharide on adipogenic differentiation of rat bone marrow mesenchymal stem cells. Food Science and Technology, 2017. 37(2): p. 303-307.

2.Liu, X.-F., J. Zhu, S.-Y. Ge, L.-J. Xia, H.-Y. Yang, Y.-T. Qian, and F.-Z. Ren, Orally administered Dendrobium officinale and its polysaccharides enhance immune functions in BALB/c mice. Natural product communications, 2011. 6(6): p. 867-870.

3.Xiang, L., C.S. Sze, T. Ng, Y. Tong, P. Shaw, C.S. Tang, and Y.K. Zhang, Polysaccharides of Dendrobium officinale inhibit TNF- $\alpha$-induced apoptosis in A-253 cell line. Inflammation Research, 2013. 62(3): p. 313-324.

4.Ng, T.B., J. Liu, J.H. Wong, X. Ye, S.C.W. Sze, Y. Tong, and K.Y. Zhang, Review of research on Dendrobium, a prized folk medicine. Applied microbiology and biotechnology, 2012. 93(5): p. 1795-1803.

5.Tang, H., T. Zhao, Y. Sheng, T. Zheng, L. Fu, and Y. Zhang, Dendrobium officinale Kimura et Migo: A Review on Its Ethnopharmacology, Phytochemistry, Pharmacology, and Industrialization. Evid Based Complement Alternat Med, 2017: p. 7436259.

6.Lin, Y., J. Li, B. Li, T. He, and Z. Chun, Effects of light quality on growth and development of protocorm-like bodies of Dendrobium officinale in vitro. Plant Cell, Tissue and Organ Culture (PCTOC), 2011. 105(3): p. 329-335.

7.Zhou, H., Q. Gao, Q. Gong, S. Qiu, J. He, J. Huang, and Y. He, First Report of Stem and Root Rot of the Medicinal Herb Dendrobium officinale (Orchidaceae) Caused by Ceratobasidium sp. AG-R in Guangxi, China. Plant Disease, 2017. 101(9): p. 1679.

8.Otero, J.T., J.D. Ackerman, and P. Bayman, Diversity and host specificity of endophytic Rhizoctonia-like fungi from tropical orchids. American Journal of Botany, 2002. 89(11): p. 18521858.

9.Tejesvi, M., M. Nalini, B. Mahesh, H. Prakash, K. Kini, H. Shetty, and V. Subbiah, New hopes from endophytic fungal secondary metabolites. Bol Soc Quím Méx, 2007. 1(1): p. 19-26.

10. Dearnaley, J.D.W., F. Martos, and M.-A. Selosse, Orchid Mycorrhizas: Molecular Ecology, Physiology, Evolution and Conservation Aspects, in Fungal Associations, B. Hock, Editor. 2012, Springer Berlin Heidelberg: Berlin, Heidelberg. p. 207-230.

11. Dressler, R. and H. Rasmussen, Terrestrial Orchids: From Seed to Mycotrophic Plant. Systematic Botany, 1996. 21: p. 625.

12. Bidartondo, M.I., The evolutionary ecology of myco-heterotrophy. New phytologist, 2005. 167(2): p. 335-352.

13. Aly, A.H., A. Debbab, and P. Proksch, Fungal endophytes: unique plant inhabitants with great promises. Applied microbiology and biotechnology, 2011. 90(6): p. 1829-1845.

14. Aly, A.H., A. Debbab, J. Kjer, and P. Proksch, Fungal endophytes from higher plants: a prolific source of phytochemicals and other bioactive natural products. Fungal diversity, 2010. 41(1): p. 1-16.

15. McCormick, M., D. Whigham, D. Sloan, and K. O'Malley, Orchid-fungus fidelity: A marriage meant to last? Ecology, 2006. 87: p. 903-11.

16. Girlanda, M., R. Segreto, D. Cafasso, H.T. Liebel, M. Rodda, E. Ercole, S. Cozzolino, G. Gebauer, and S. Perotto, Mediterranean meadow photosynthetic orchids feature partial mycoeterotrophy and specific mycorrhizal associations. American journal of botany, 2011. 98: p. 11481163.

17. Xing, X., X. Ma, Z. Deng, J. Chen, F. Wu, and S. Guo, Specificity and preference of 
383

384

385

386

387

388

389

390

391

392

393

394

395

396

397

398

399

400

401

402

403

404

405

406

407

408

409

410

411

412

413

414

415

416

417

418

419

420

421

422

423

424

425

426

427

428

mycorrhizal associations in two species of the genus Dendrobium (Orchidaceae). Mycorrhiza, 2013. 23(4): p. 317-324.

18. Klironomos, J.N., Variation in plant response to native and exotic arbuscular mycorrhizal fungi. Ecology, 2003. 84(9): p. 2292-2301.

19. Raju, P., R. Clark, J. Ellis, and J. Maranville, Effects of species of VA-mycorrhizal fungi on growth and mineral uptake of sorghum at different temperatures. Plant and soil, 1990. 121(2): p. $165-170$.

20. Kosuta, S., M. Chabaud, G. Lougnon, C. Gough, J. Dénarié, D.G. Barker, and G. Bécard, A Diffusible Factor from Arbuscular Mycorrhizal Fungi Induces Symbiosis-Specific MtENOD11 Expression in Roots of Medicago truncatula. Plant physiology, 2003. 131(3): p. 952-962.

21. Jacquemyn, H., K.J. Duffy, and M.-A. Selosse, Biogeography of orchid mycorrhizas, in Biogeography of mycorrhizal symbiosis. 2017, Springer. p. 159-177.

22. Hossain, M.M., R. Kant, P.T. Van, B. Winarto, S. Zeng, and J.A. Teixeira da Silva, The application of biotechnology to orchids. Critical Reviews in Plant Sciences, 2013. 32(2): p. 69139.

23. Bodah, E.T., Root rot diseases in plants: a review of common causal agents and management strategies. Agri Res \& Tech: Open Access J, 2017. 5(3): p. 555661.

24.Zhang, Y., B. Lin, M. Zou, J. Liang, and H. Hu, First Report of Fusarium Wilt of Dendrobium officinale Caused by Fusarium oxysporum in China. Plant Disease, 2017. 101(6): p. 1039-1039.

25. Sardrood, B.P. and E.M. Goltapeh, Effect of Agricultural Chemicals and Organic amendments on biological control fungi, in Sustainable Agriculture Reviews 31. 2018, Springer. p. 217-359.

26. Mahanty, T., S. Bhattacharjee, M. Goswami, P. Bhattacharyya, B. Das, A. Ghosh, and P. Tribedi, Biofertilizers: a potential approach for sustainable agriculture development. Environmental Science and Pollution Research, 2017. 24(4): p. 3315-3335.

27. Singh, J.S., V.C. Pandey, and D. Singh, Efficient soil microorganisms: a new dimension for sustainable agriculture and environmental development. Agriculture, ecosystems \& environment, 2011. 140(3-4): p. 339-353.

28. Guo, S.T., W.U. Jian-Rong, H.U. Jun, H.G. Yang, L.U. Lu, and F. Liu, Symbiotic seed germination of Cymbidium mastersii Griff.ex Lindl. Journal of Yunnan University(Natural Sciences Edition), 2012. 34(3): p. 348-355.

29. Wu, J., H. Ma, X. Xu, N. Qiao, S. Guo, F. Liu, D. Zhang, and L. Zhou, Mycorrhizas alter nitrogen acquisition by the terrestrial orchid Cymbidium goeringii. Annals of Botany, 2013(6): p. 1181-1187.

30. Dong, F., Screen several orchid mycorrhizal fungi and preliminary test on the seed germination condition. 2008, Beijing Forestry University.

31. Jin, H., Z.X. Xu, H. Chen, and S.F. Han, Localization and changes of activity of acid phosphatase in mycorhiza of Cymbidium hookerianum. Journal of Beijing Forestry University, 2007. 29(4): p. 156-160.

32. Dijk, E. and N.D. Eck, Effects of mycorrhizal fungi on in vitro nitrogen response of some Dutch indigenous orchid species. Canadian Journal of Botany, 1995. 73(8): p. 1203-1211.

33. Murashige, T. and F. Skoog, A revised medium for rapid growth and bioassay with tobacco tissue cultures. Physiologia Plantarum, 1962. 15(3): p. 473-497.

34. Gilbertson, R.L., The use of dry-leaf inoculum for establishment of common bacterial blight of beans. Plant Disease, 1988. 72(5): p. 385-389. 
436

437

438

439

440

441

442

443

444

445

446

447

448

449

450

451

452

453

454

455

456

457

458

459

460

461

462

463

464

465

466

467

468

469

470

471

472

473

474

35. Munro, R.C., J. Wilson, J. Jefwa, and K.W. Mbuthia, A low-cost method of mycorrhizal inoculation improves growth of Acacia tortilis seedlings in the nursery. Forest Ecology and Management, 1999. 113(1): p. 51-56.

36. Şükran, D., T. GÜNEŞ, and R. Sivaci, Spectrophotometric determination of chlorophyllA, B and total carotenoid contents of some algae species using different solvents. Turkish Journal of Botany, 1998. 22(1): p. 13-18.

37. White, T., T. Bruns, S. Lee, F. Taylor, T. White, S.H. Lee, L. Taylor, and J. Shawetaylor, Amplification and direct sequencing of fungal ribosomal RNA genes for phylogenetics. PCR protocols: a guide to methods and applications. 1990: p. 315-322.

38. Phylogenetic Diversity and Microsphere Array-Based Genotyping of Human Pathogenic Fusaria, Including Isolates from the Multistate Contact Lens-Associated U.S. Keratitis Outbreaks of 2005 and 2006. Journal of Clinical Microbiology, 2007. 45(7): p. 2235-2248.

39. A, G.H.S., J.M.S. B, H.J. C, and J.W.S. A, A multi-gene phylogeny of Clavicipitaceae (Ascomycota, Fungi): identification of localized incongruence using a combinational bootstrap approach. Molecular Phylogenetics \& Evolution, 2007. 44(3): p. 1204-1223.

40. Meyer, M. and M.K. Hausbeck, Using Soil-Applied Fungicides to Manage Phytophthora Crown and Root Rot on Summer Squash. Plant Disease, 2013. 97(1): p. 107-112.

41. Dubey, M.K., A. Broberg, F.J. Dan, and M. Karlsson, Role of the methylcitrate cycle in growth, antagonism and induction of systemic defence responses in the fungal biocontrol agent Trichoderma atroviride. Microbiology-sgm, 2013. 159(Pt_12): p. 2492-2500.

42. Dubey, M.K., F.J. Dan, and M. Karlsson, An ATP-binding cassette pleiotropic drug transporter protein is required for xenobiotic tolerance and antagonism in the fungal biocontrol agent Clonostachys rosea. Mol Plant Microbe Interact, 2014. 27(7).

43. Chen, J., H. Wang, and S.X. Guo, Isolation and identification of endophytic and mycorrhizal fungi from seeds and roots of Dendrobium (Orchidaceae). Mycorrhiza, 2012. 22(4): p. 297-307.

44. Vega, F.E., The use of fungal entomopathogens as endophytes in biological control: a review. Mycologia, 2018. 110(1): p. 4-30.

45. Shahzad, R., A.L. Khan, S. Bilal, S. Asaf, and I.-J. Lee, What is there in seeds? Vertically transmitted endophytic resources for sustainable improvement in plant growth. Frontiers in plant science, 2018. 9: p. 24.

46. Brundrett and C. Mark, Scientific approaches to Australian temperate terrestrial orchid conservation. Australian Journal of Botany, 2007. 55(3): p. 293-307.

47. Jacquemyn, H., M. Waud, V. Merckx, B. Lievens, and R. Brys, Mycorrhizal diversity, seed germination and long-term changes in population size across nine populations of the terrestrial orchid Neottia ovata. Molecular Ecology, 2015. 24(13): p. 3269-3280.

48.Zi, X.M., C.L. Sheng, U.M. Goodale, S.C. Shao, and J.Y. Gao, In situ seed baiting to isolate germination-enhancing fungi for an epiphytic orchid, Dendrobium aphyllum (Orchidaceae). Mycorrhiza, 2014. 24(7): p. 487-499.

49. Ohashi-Kaneko, K., K. Fujiwara, Y. Kimura, and K. Kurata, Effect of Blue-Light PPFD Percentage in Red and Blue LED Low-Light Irradiation during Storage on the Contents of Chlorophyll and Rubisco in Grafted Tomato Plug Seedlings. Environment Control in Biology, 2010. 44(4): p. 309-314.

50. Kirschner, R., Response of Arbuscular Mycorrhizal Fungi on Growth and Chlorophyll Content of Three Varieties of Gossypium herbaceum L. Plant Pathology \& Quarantine, 2013. 3: p. 54-57.

Peer) reviewing PDF | (2021:03:58984:2:0:NEW 14 Oct 2021) 
475

476

477

478

479

480

481

482

483

484

485

486

487

488

489

490

491

492

493

494

495

496

497

498

499

500

501

502

503

504

505

506

507

508

509

510

511

512

513

514

515

516

517

518

519

520

51. Rajasekaran, S., S.M. Nagarajan, K. Arumugam, R. Saravanamuthu, and S. Balamurugan, Effect of dual inoculation (Rhizobium and AM fungi) on chlorophyll content of Arachis hypogaea L. CV. TMV-2. Plant Archives, 2006. 6(2): p. 671-672.

52. Cameron, D., J. Irene, J.R. Leake, and D.J. Read, Mycorrhizal Acquisition of Inorganic Phosphorus by the Green-leaved Terrestrial Orchid Goodyera repens. Ann Bot, 2007(5): p. 831834.

53. Alexander, C. and I. Hadley, Phosphate uptake by in relation to mycorrhizal infection. New Phytologist, 1984. 97(3): p. 401-411.

54. Plassard, C., A. Becquer, and K. Garcia, Phosphorus transport in mycorrhiza: how far are we? Trends in plant science, 2019. 24(9): p. 794-801.

55. Siti, N., N.D. Swarts, K.W. Dixon, L. Hans, and D.J. Merritt, Variation in nutrientacquisition patterns by mycorrhizal fungi of rare and common orchids explains diversification in a global biodiversity hotspot. Annals of Botany, 2013(6): p. 1233-1241.

56. Liu, C.Y., The changes of the vitality of Armillaria mellea and the histochemical localization of some enzymes in the hyphae digested period of gastrodia elata. Acta Botanica Sinica, 1982. 24(4): p. 307-311.

57. Conn, C. and J. Dighton, Litter quality influences on decomposition, ectomycorrhizal community structure and mycorrhizal root surface acid phosphatase activity. Soil Biology \& Biochemistry, 2000. 32(4): p. 489-496.

58. Zaidi, A., M.S. Khan, M. Ahemad, M. Oves, and P. Wani, Recent advances in plant growth promotion by phosphate-solubilizing microbes. Microbial strategies for crop improvement, 2009: p. 23-50.

59. Smith, S.E., E. Facelli, S. Pope, and F.A. Smith, Plant performance in stressful environments: interpreting new and established knowledge of the roles of arbuscular mycorrhizas. Plant and Soil, 2010. 326(1-2): p. 3-20.

60. Singh, L.P., S.S. Gill, and N. Tuteja, Unraveling the role of fungal symbionts in plant abiotic stress tolerance. Plant signaling \& behavior, 2011. 6(2): p. 175-191.

61. Rapparini, F. and J. Peñuelas, Mycorrhizal fungi to alleviate drought stress on plant growth, in Use of Microbes for the Alleviation of Soil Stresses, Volume 1. 2014, Springer. p. 2142.

62. Wang, R.Y., B. Gao, X.H. Li, J. Ma, and S.L. Chen, First Report of Fusarium solani Causing Fusarium Root Rot and Stem Canker on Storage Roots of Sweet Potato in China. Plant Disease, 2014. 98(1): p. 160-160.

63. Latiffah, Z., M.Z.N. Hayati, S. Baharuddin, and Z. Maziah, Identification and Pathogenicity of Fusarium Species Associated with Root Rot and Stem Rot of Dendrobium. Asian Journal of Plant Pathology, 2010. 3(1): p. 14-21.

64. Mesterhazy, A., M. Lemmens, and L.M. Reid, Breeding for resistance to ear rots caused by Fusarium spp. in maize-a review. Plant Breeding, 2012. 131(1): p. 1-19.

65. Nemec, S. and R.M. Zablotowicz, Effect of soil temperature on root rot of rough lemon caused by Fusarium solani. Mycopathologia, 1981. 76(3): p. 185-190.

66. Bohra, B. and K. Mathur, Biocontrol Agents and Neem Formulations for Suppression of Fusarium solani Root Rot in Soybean. Journal of Mycology \& Plant Pathology, 2004. 34(2): p. 408-409.

67. Lee, T.V.D., H. Zhang, A.V. Diepeningen, and C. Waalwijk, Biogeography of Fusarium graminearum species complex and chemotypes: a review. Food Additives \& Contaminants: Part A, 2015. 32(4): p. 453-460. 
523

524

525

526

527

528

529

530

531

532

533

534

535

536

537

538

539

540

541

542

543

544

545

546

547

548

549

550

551

552

553

554

555

556

557

558

559

560

561

562

563

564

565

566

68. Kikot, G.E., R.A. Hours, and T.M. Alconada, Contribution of cell wall degrading enzymes to pathogenesis of Fusarium graminearum: a review. Journal of Basic Microbiology, 2009. 49(3): p. 231-241.

69. Swett, C.S. and J.Y. Uchida, Characterization of Fusarium diseases on commercially grown orchids in Hawaii. Plant Pathology, 2015. 64(3): p. 648-654.

70. Latiffah, N. Hayati, Z. M., Baharuddin, and Maziah, Vegetative Compatibility Group of Fusarium species Associated with Root and Stem Rot of Orchid. Malaysian Journal of Microbiology, 2008. 4(1): p. 49-52.

71. Srivastava, S., Characterization and management of different Fusarium species associated with orchids cultivated in Hawai'i. Dissertations \& Theses - Gradworks, 2014. 76(01): p. 131.

72. Lu, Y., C. Su, N.V. Solis, S.G. Filler, and H. Liu, Synergistic regulation of hyphal elongation by hypoxia, $\mathrm{CO} 2$, and nutrient conditions controls the virulence of Candida albicans. Cell host \& microbe, 2013. 14(5): p. 499-509.

73. Fernandez, J., M. Marroquin-Guzman, and R.A. Wilson, Mechanisms of nutrient acquisition and utilization during fungal infections of leaves. Annual review of phytopathology, 2014. 52: p. 155-174.

74. Wanderley, M., J.M.W.D. Neto, J. Filho, C. Lima, and A. Porto, Collagenolytic enzymes produced by fungi: a systematic review. Brazilian Journal of Microbiology, 2017. 48(1): p. 13-24.

75. Krywolap, G.N., L.F. Grand, and L. Jr, The natural occurrence of an antibiotic in the mycorrhizal fungus Cenococcum graniforme. Canadian Journal of Microbiology, 2011. 10(3): p. 323-328.

76. Santoro, T. and L.E. Casida, Elaboration of antibiotics by Boletus luteus and certain other mycorrhizal fungi. Canadian Journal of Microbiology, 1962. 8(1): p. 43-48.

77. O’Brien, P.A., Biological control of plant diseases. Australasian Plant Pathology, 2017. 46(4): p. 293-304.

78. Toghueo, R.M.K., P. Eke, Í. Zabalgogeazcoa, B.R.V. de Aldana, L.W. Nana, and F.F. Boyom, Biocontrol and growth enhancement potential of two endophytic Trichoderma spp. from Terminalia catappa against the causative agent of Common Bean Root Rot (Fusarium solani). Biological Control, 2016. 96: p. 8-20.

79. Egamberdieva, D., S.J. Wirth, V.V. Shurigin, A. Hashem, and E.F. Abd_Allah, Endophytic bacteria improve plant growth, symbiotic performance of chickpea (Cicer arietinum L.) and induce suppression of root rot caused by Fusarium solani under salt stress. Frontiers in Microbiology, 2017. 8: p. 1887.

80. Jaber, L.R. and B.H. Ownley, Can we use entomopathogenic fungi as endophytes for dual biological control of insect pests and plant pathogens? Biological control, 2018. 116: p. 36-45.

81. Dell, B., Role of mycorrhizal fungi in ecosystems. CMU. Journal, 2002. 1(1): p. 47-60.

82. Gao, F., C. Dai, and X. Liu, Mechanisms of fungal endophytes in plant protection against pathogens. Afr J Microbiol Res, 2010. 4(13): p. 1346-1351.

83. Mittler, R. and E. Blumwald, Genetic Engineering for Modern Agriculture: Challenges and Perspectives. Annual Review of Plant Biology, 2010. 63(1): p. 443-462.

84. MacDonald, J., and D., Effect of Salinity Stress on the Development of Phytophthora Root Rot of Chrysanthemum. Phytopathology, 1982. 72(2): p. 214-214.

85. Pandey, P., R. Sinha, K.S. Mysore, and M. Senthil-Kumar, Impact of Concurrent Drought Stress and Pathogen Infection on Plants, in Combined Stresses in Plants: Physiological, Molecular, and Biochemical Aspects, R. Mahalingam, Editor. 2015, Springer International Publishing: Cham. p. 203-222. 


\section{Figure 1}

Mycorrhizal fungi promote the growth of tissue culture seedlings of Dendrobium officinale.

GDB254, MLX102, GS222, and GDB162 were inoculated into D. officinale seedlings for 60 days and were associated with increased weight acquisition compared to that in the control group (CK).
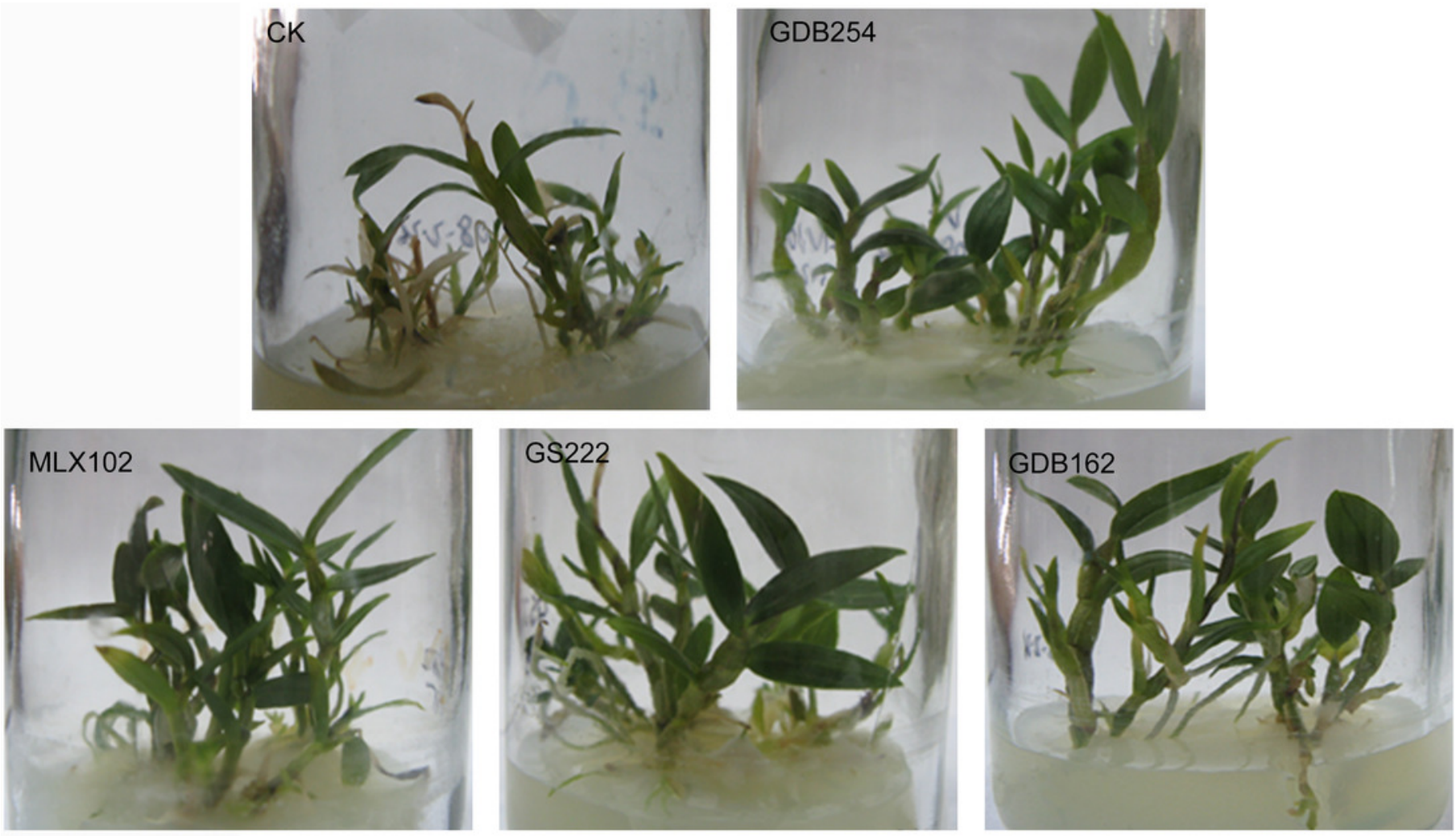
Figure 2

Mycorrhizal fungi promote the growth of tissue culture seedlings of Dendrobium officinale.

The growth-promoting effects of GDB254, MLX102, GS222, and GDB162 on D. officinale tissue culture seedlings after 60 days. (A), (B), (C), and (D) show the fresh weight, chlorophyll content, stem polysaccharide levels, and root acid phosphatase activity of $D$. officinale, respectively; the values of the parameters increased after symbiotic growth with different mycorrhizal fungi. Letters over the bars indicate significant differences at the $5 \%$ level.

A
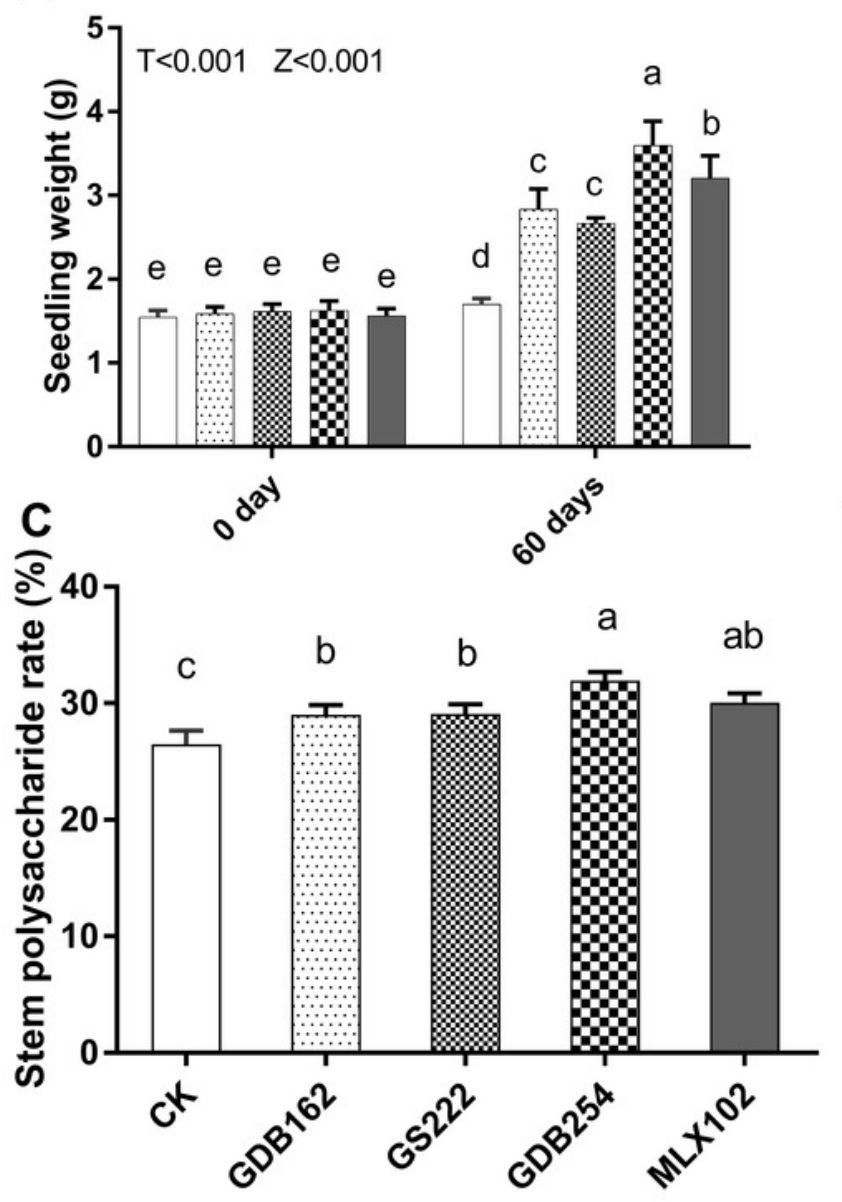

B

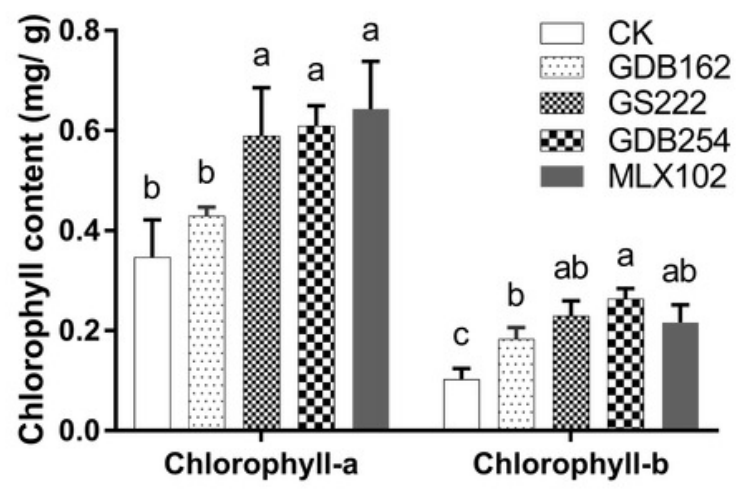

D

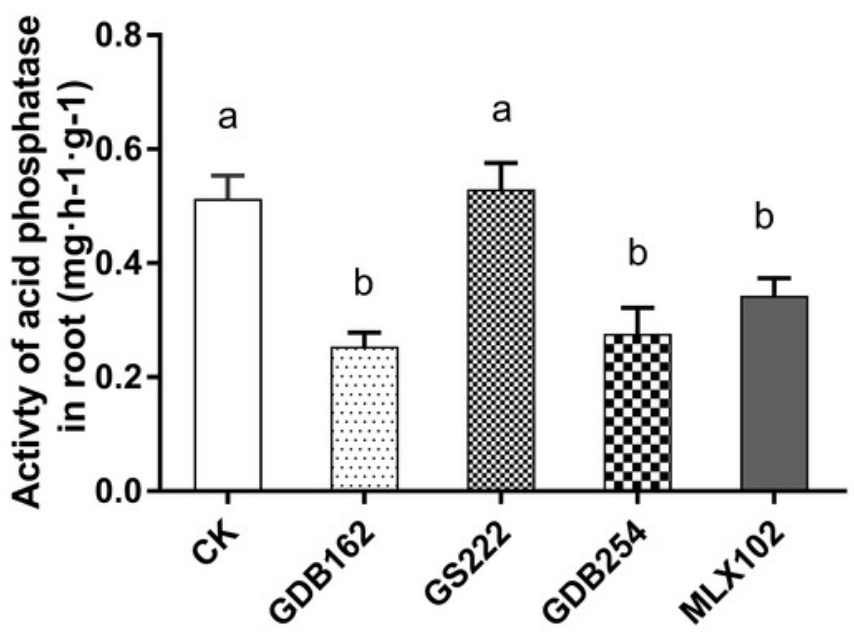


Figure 3

Growth-promoting effects of mycorrhizal fungi on Dendrobium officinale cultivated in pots.

(A) Effects of mycorrhizal fungi on the survival rate after transplantation from the culture bottles to flower pots. (B, C, and D) Growth-promoting effects of various mycorrhizal fungi on (B) the stem diameter, (C) stem length, and (D) leaf number of the plants grown in pots. Different letters over the bars indicate significant differences at the $5 \%$ level.

A

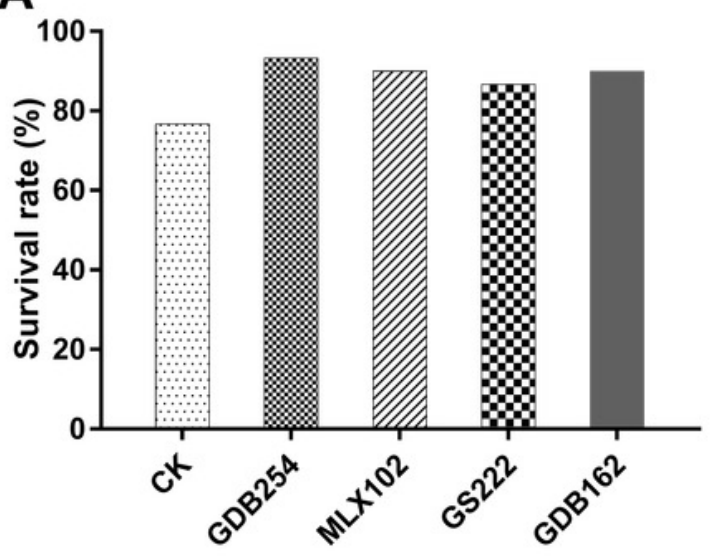

C

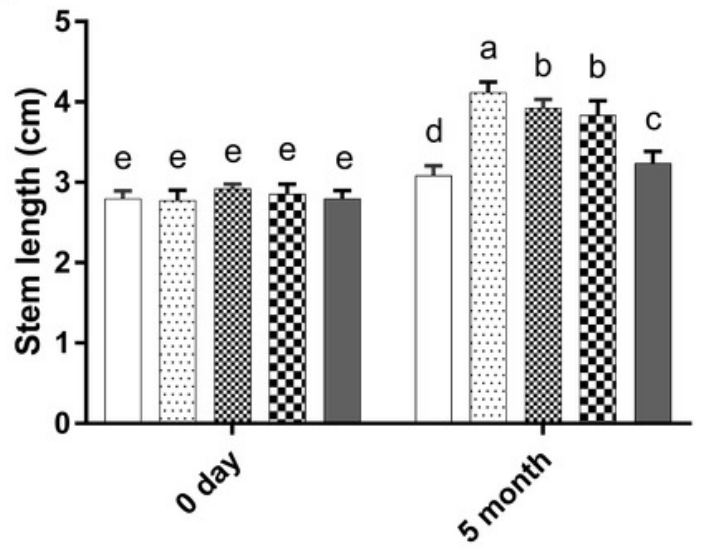

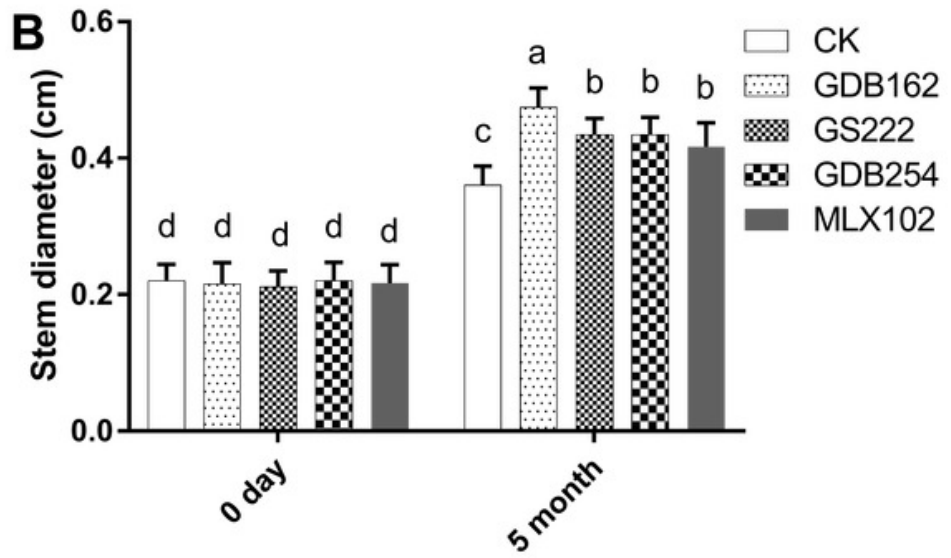

D

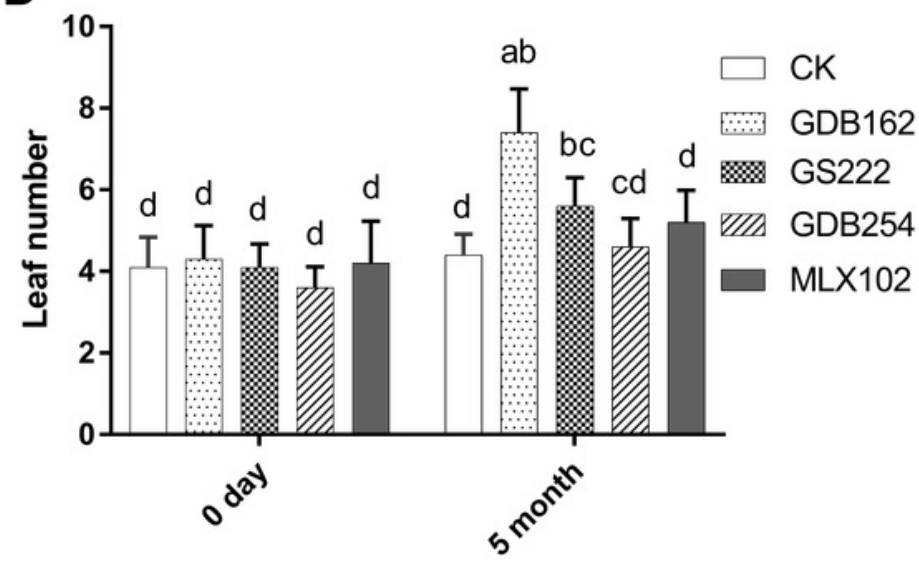


Figure 4

The effect of mycorrhizal fungi on the drought resistance of $D$. officinale.

(A) The effect of mycorrhizal fungi on the survival rate under drought conditions. (B) The effect of mycorrhizal fungi on the MDA contents of $D$. officinale under various drought conditions.
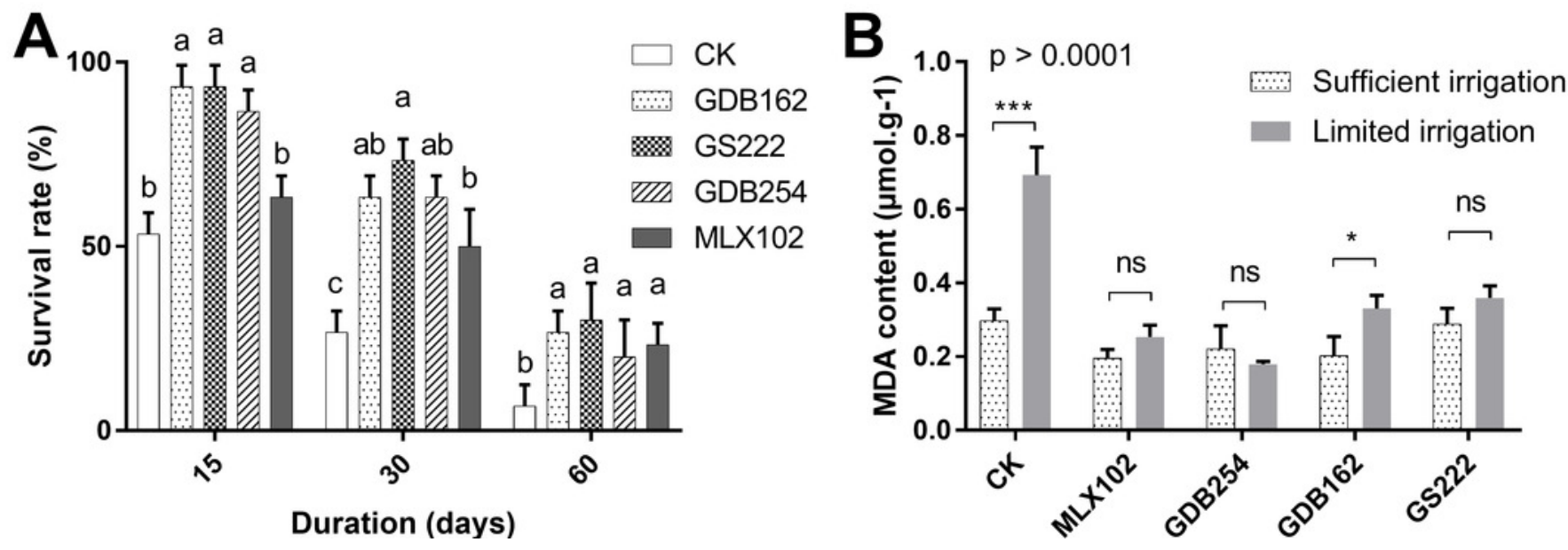


\section{Figure 5}

Morphology and microstructure of pathogenic fungi.

Morphology of TS1 (A) and TS2 (D) strains grown on PSA. (B), (C) are the microscopic morphology of TS1. (E), (F) are the microscopic morphology of TS2. Colonies of TS1 were formed after 3 days of growth on PSA medium at $28^{\circ} \mathrm{C}$
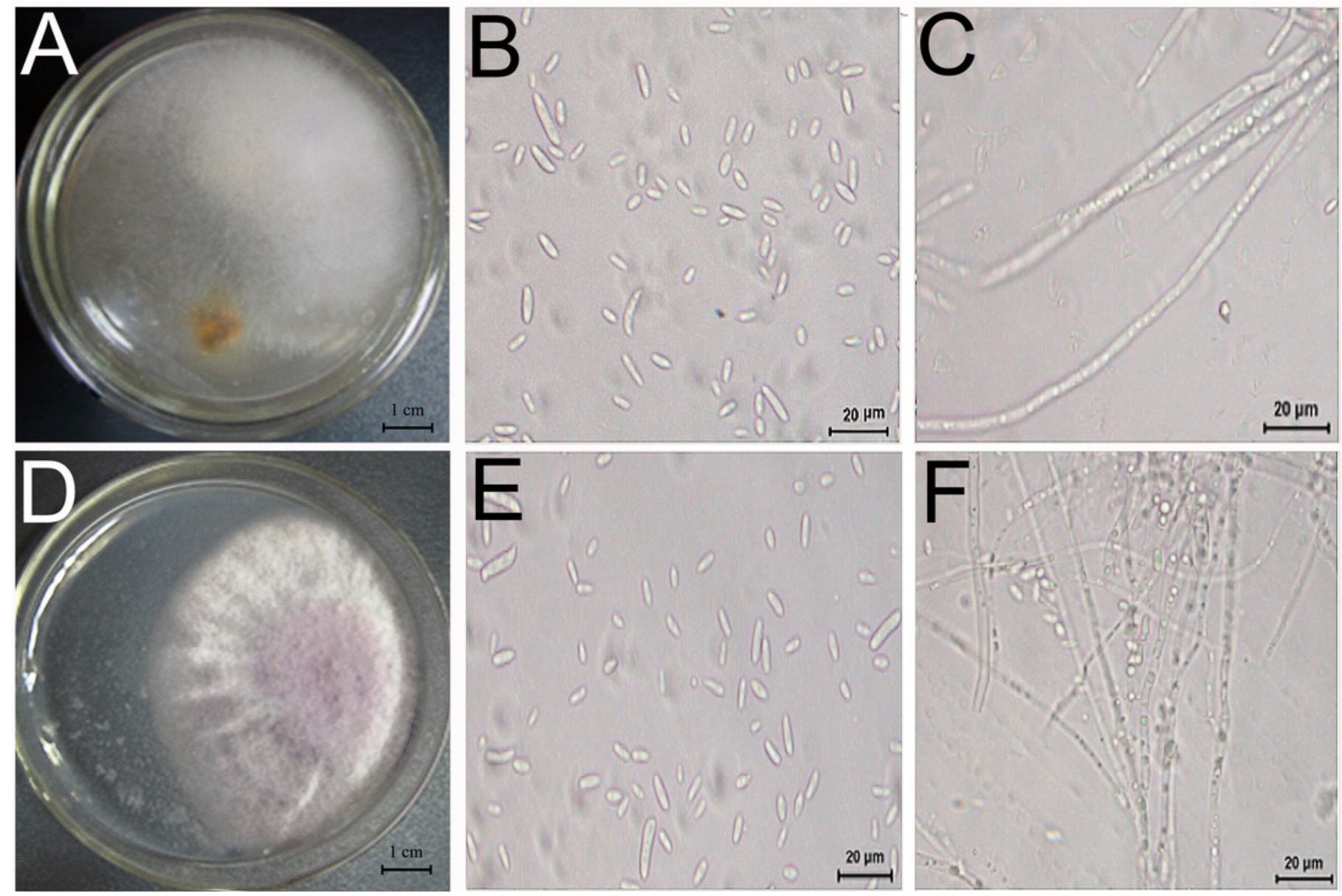
Figure 6

TS1 and TS2 strains all showed pathogenicity to Dendrobium officinale.

(A) is the control group, which was not inoculated with pathogenic fungus. TS1 (B) and TS2

(C) were incubated with $D$. officinale seedlings. The solid arrows point to the rot root and hollow arrow point to healthy root.
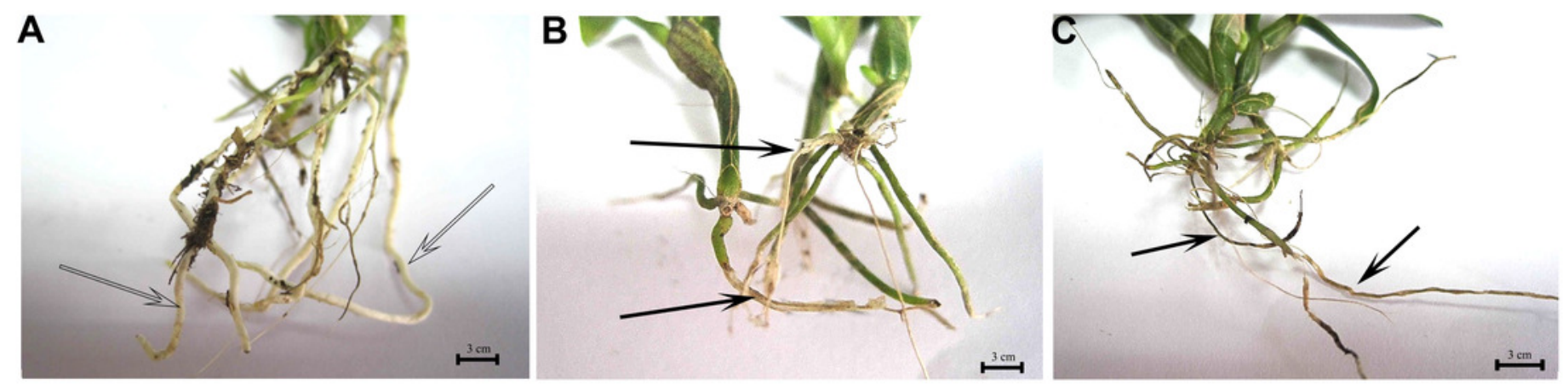


\section{Figure 7}

The competitive effect of mycorrhizal fungi with pathogenic fungi.

The mycorrhizal fungi (GDB254, GS222 MLX 102, and GDP162) competed with the pathogenic fungi (TS1 and TS2) by means of orthogonal experimental design, and the growth curves of the pathogenic fungi were plotted and analysed. (A-D) shows the mycorrhizal fungus competing with TS1 and (E-H) shows the mycorrhizal fungus competing with TS2.
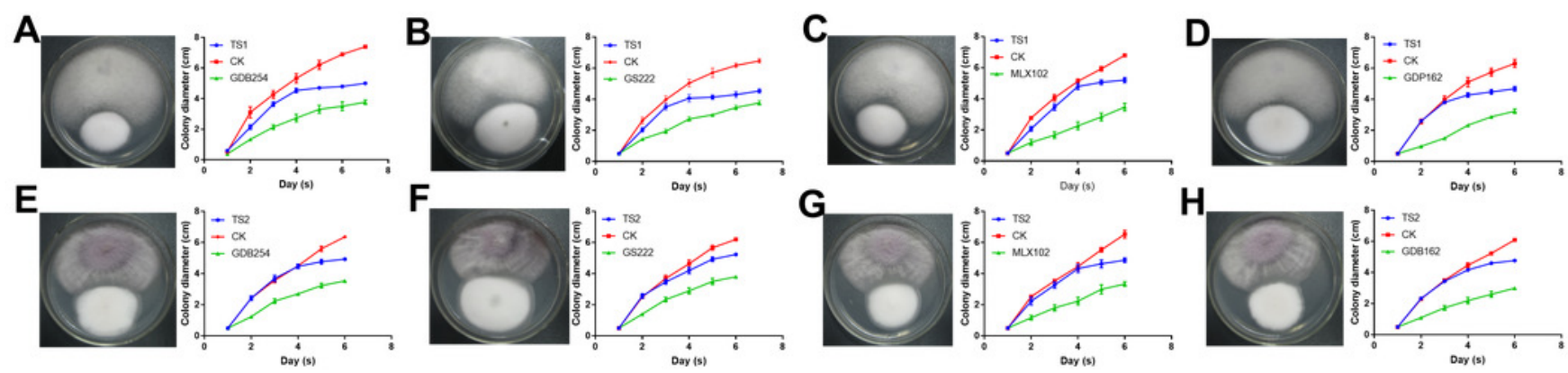
Figure 8

Mycorrhizal fungi increase the resistance of Dendrobium officinale.

Mycorrhizal fungi interacting with $D$. officinale could increase its disease resistance and thus improve its survival rate on DE medium (A), inhibit root rot (B) and increase fresh weight yield (C) under disease stress.
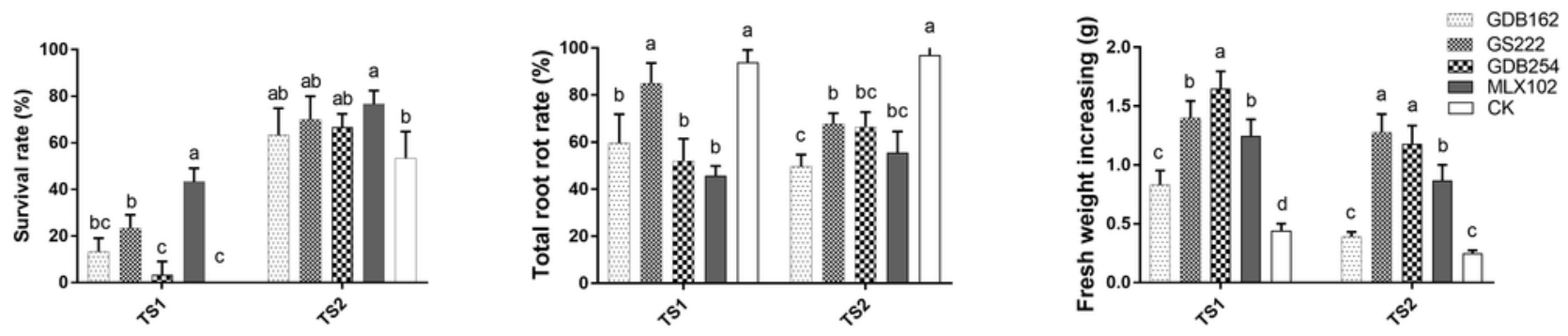